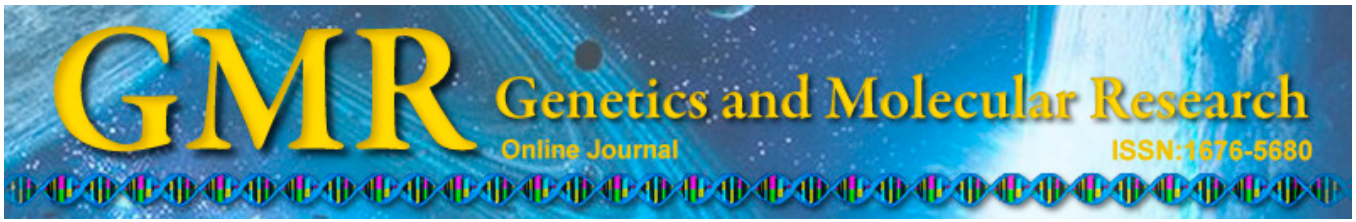

\title{
Changes in hippocampal ultrastructure and vimentin expression in rhesus monkeys following selective deep hypothermia and blood occlusion
}

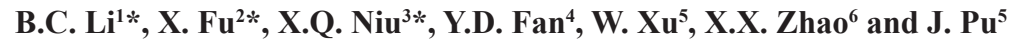 \\ ${ }^{1}$ Department of Neurosurgery, PuEr Municipal Hospital, PuEr, \\ Yunnan Province, China \\ ${ }^{2}$ Department of Neurosurgery, Suzhou Municipal Hospital, Suzhou, \\ Jiangsu Province, China \\ ${ }^{3}$ Department of Pneumology, \\ Second Affiliated Hospital of KunMing Medical University, \\ KunMing, Yunnan Province, China \\ ${ }^{4}$ Department of Neurosurgery, \\ Third Affiliated Hospital of KunMing Medical University, \\ KunMing, Yunnan Province, China \\ ${ }^{5}$ Department of Neurosurgery, \\ Second Affiliated Hospital of KunMing Medical University, KunMing, \\ Yunnan Province, China \\ ${ }^{6}$ Department of Radiology, Second Affiliated Hospital of KunMing Medical \\ University, KunMing, Yunnam Province, China \\ *These authors contributed equally to this study. \\ Corresponding author: J. Pu \\ E-mail: pujun303@aliyun.com
}

Genet. Mol. Res. 14 (1): 651-658 (2015)

Received April 25, 2014

Accepted October 7, 2014

Published January 30, 2015

DOI http://dx.doi.org/10.4238/2015.January.30.7

ABSTRACT. Previous studies have shown that selective cerebral profound hypothermia combined with antegrade cerebral perfusion can improve resistance to cerebral hypoxia-ischemia in monkeys. The aim 
of this study was to observe the effect of selective cerebral profound hypothermia on the ultrastructure and vimentin expression in monkey hippocampi after severe cerebral ischemia. Eight healthy adult rhesus monkeys were randomly divided into two groups: profound hypothermia $(\mathrm{N}=5)$ and normothermia $(\mathrm{N}=3)$. Monkeys in the profound hypothermia group underwent bilateral carotid artery and jugular vein occlusion for 10 minutes at room temperature. Ringer's solution at $4{ }^{\circ} \mathrm{C}$ was then perfused through the right internal carotid artery and out of the right jugular vein, maintaining the brain temperature below $18^{\circ} \mathrm{C}$. Sixty minutes later, cerebral blood flow was restored. The normothermia group underwent all procedures with the exception that the Ringer's solution was $37^{\circ} \mathrm{C}$ during perfusion. All animals in the profound hypothermia group were successfully resuscitated. No significant abnormalities of hippocampal morphology or ultrastructure were observed. In contrast, no monkeys were alive after perfusion in the normothermia group and they had abnormal hippocampal morphology and ultrastructure to different extents. Vimentin expression in the hippocampus was significantly lower in the profound hypothermia group $(47.88 \% \pm 1.66)$ than the normothermia group $(79.51 \% \pm 1.00 ; \mathrm{P}<0.01)$. We conclude that selective cerebral profound hypothermia following 10-min occlusion of the bilateral common carotid arteries was able to downregulate vimentin expression in the hippocampus and protect it from severe cerebral ischemia.

Key words: Ultrastructure; Vimentin; Hippocampus; Blood occlusion; Hypothermia

\section{INTRODUCTION}

Although only $2 \%$ of whole body mass, normal human cerebrum receives $15 \%$ of cardiac blood output and uses $20 \%$ of systemic oxygen consumption, indicating the considerable demand for blood and oxygen by the cerebrum. At normal temperatures, the ischemia endurance time of the human cerebrum is $5-8 \mathrm{~min}$; consciousness is lost by $10 \mathrm{~s}$ after absolute blood occlusion. Thus, the cerebrum has low endurance and is very sensitive to ischemia and hypoxia (Yao, 2001). Complex intracranial space occupying lesions in neurosurgery, such as huge complex intracranial aneurysms of the posterior circulation or huge complex intracranial vascular malformations, require the induction of a cerebral blood occlusion to insure surgery success and reduce surgical complications. However, many researchers have studied protection of the cerebrum following cerebral occlusion as it is an important factor that influences prognosis (Elmistekawy and Rubens, 2011; Nakamura et al., 2011).

Hypothermia, which slows down the cerebral metabolism and reduces cerebral hypoxia, is deemed as one of the most efficient and promising protective measures against cerebral ischemia (Ginsberg, 2008; Mracek, 2010). Currently, deep hypothermic systemic circulatory arrest technology is available in clinical neurosurgery but has many disadvantages that mean the blood occlusion safe time cannot meet the clinical surgery operation time requirements; therefore, it can easily induce complications, such as blood viscosity, blood coagulation, arrhythmia, and respiratory failure (Cronberg et al., 2009). Previous study results have indicated that deep hypothermia 
with selective antegrade cerebral perfusion can improve cerebral endurance to ischemia and hypoxia in the cerebrum of rhesus monkeys, extend the safe time of blood occlusion, and avoid various systemic deep hypothermia complications (Yao, 2001; Elmistekawy and Rubens, 2011). In this study, the effectiveness and safety of selective cerebral deep hypothermic protection technology is further discussed by observing the change in hippocampal ultrastructure and vimentin expression in rhesus monkeys after recovery from selective deep hypothermia and blood occlusion.

\section{MATERIAL AND METHODS}

\section{Design}

This experiment was designed as a comparative observation animal experiment and took place at the Experimental Animal Center of Kunming Medical College on February 7, 2011.

\section{Materials}

\section{Experimental animals}

Eight healthy adult rhesus monkey were purchased from the experimental center of Kunming Animal Institute, Chinese Academy of Science, with no limit on gender and with a body mass between $6.9-11.2 \mathrm{~kg}$ (mean body mass $=8.1 \mathrm{~kg}$ ).

\section{Agent and Instrument}

TH-5 cerebrum temperature monitor was purchased from Virginia University, USA. The JEM-100CX transmission electron microscope was purchased from Japan Electronic Corporation. Vimentin primary antibody was purchased from Wuhan Boster Biological Technology (China) and the secondary antibody was purchased from DAKO (Denmark).

\section{Methods}

\section{Cerebrum ischemia, hypoxia model establishment, and experimental grouping}

Rhesus monkeys were housed under constant temperature, humidity, and ventilation for one week prior to random division into the deep hypothermia group $(\mathrm{N}=5)$ or normal temperature group $(\mathrm{N}=3)$. The deep hypothermia group was anesthetized with propofol (AstraZeneca, USA, $2.0 \mathrm{mg} / \mathrm{kg}$ ). Once anesthetization had been confirmed, local extracorporeal circulation was established and right frontal lobes of the rhesus monkeys were inserted with needle shaped probe to connect to the cerebrum temperature monitor. All rhesus monkeys were heparinized systemically before the temperature was lowered. The bilateral common carotid arteries and jugular veins were occluded to induce ischemia at a normal temperature for 10 min. Following this, Ringer's solution at $4^{\circ} \mathrm{C}$ was transfused into right internal carotid arteries with a transfusion speed of $10 \mathrm{~mL} \cdot \mathrm{kg}^{-1} \cdot \mathrm{min}^{-1}$ ) to induce deep hypothermia. The perfusate was returned through right internal jugular vein and into to systemic circulating blood through the left femoral vein. Water retention was excluded using an ultrafilter with rewarming to $38^{\circ} \mathrm{C}$ before returning to the left femoral vein. Once the cerebrum temperature lowered to $18^{\circ} \mathrm{C}$, the transfusion speed was slowed down to maintain the cerebrum temperature $\leq 18^{\circ} \mathrm{C}$. 
Hypothermic transfusion was stopped after $60 \mathrm{~min}$ and the common carotid arteries and jugular veins were opened to rewarm the cerebrum temperature slowly. Surplus water in the body was filtered, and blood that remained in the ultrafilter was transfused back slowly. The normal temperature group underwent the same procedure as the deep hypothermia, with the exception that the perfusate was Ringer's solution at $37^{\circ} \mathrm{C}$.

\section{Sampling and light microscope observation}

Rhesus monkeys in the deep hypothermia group were housed after the procedure for a further 12 weeks and then terminally anesthetized for tissue extraction. Whole cerebrum tissue was removed by craniotomy. A section of the right hippocampus was sub dissected and fixed with $3 \%$ glutaraldehyde $10 \mathrm{~g} / \mathrm{L}$ osmium tetroxide for electron microscope observation and the left hippocampus was fixed with a $10 \%$ formaldehyde fixative. Rhesus monkeys in normal temperature group died during the perfusion; therefore, a section of their right hippocampus was removed directly and fixed with $3 \%$ glutaraldehyde $10 \mathrm{~g} / \mathrm{L}$ osmium tetroxide for electron microscope observation. The left hippocampus was fixed with a $10 \%$ formaldehyde fixative. Once dehydrated with gradient ethanol, all samples were embedded in paraffin wax, sectioned, and stained using hematoxylin-eosin. Hippocampus tissue was observed by light microscope.

\section{Electron microscope observation}

Hippocampus tissue was fixed by $3 \%$ glutaraldehyde $10 \mathrm{~g} / \mathrm{L}$ osmium tetroxide, dehydrated from $30-100 \%$ gradient acetone, embedded in epoxy resin, sectioned using a LKV-V ultramicrotome $(50 \mathrm{~nm})$ and double-stained using uranyl acetate/lead citrate. The hippocampal ultrastructure was observed by JEM-100CX transmission electron microscope.

\section{Immunohistochemistry}

Cerebrum tissue was fixed in $40 \mathrm{~g} / \mathrm{L}$ paraformaldehyde. Paraffin sections were dewaxed, hydrated, underwent antigen retrieval, and treated with $3 \%$ peroxide to eliminate endogenous peroxidase activity. Following this, sections were incubated in normal lowlenthal serum to block non-specific binding. Sections were incubated with the primary antibody against vimetin $(1: 500)$ overnight at $4{ }^{\circ} \mathrm{C}$. A negative control of phosphate-buffered saline (PBS) was used, while the positive tissue was a positive control. After PBS washes, sections were incubated with streptomycete antibiotin protein-peroxidase for $15 \mathrm{~min}$ at room temperature, stained with benzidine, and finally stained by hematoxylin. The number of positive stained cells and the total cell number was counted in region of the section using the nerve cell counting method by Cai et al. (1994). The positive cell number was calculated as a positive cell rate, which was equal to the percentage positive cell number divided by total cell number.

\section{Major observation indicators}

Changes in ultrastructure and expression of vimentin were assessed in the hippocampus of the two groups.

\section{Statistical analysis}

All data are reported as means \pm standard error (SE) and compared with the Student 
$t$-test using SPSS 13.0 (IBM).

\section{RESULTS}

The selective cerebral deep hypothermic ischemia recovery model in rhesus monkeys was successfully established. Selective deep hypothermia with antegrade cerebral perfusion after cerebral ischemia was established successfully in all 5 rhesus monkeys, with successful survival after recovery. The 3 rhesus monkeys in the normal temperature group did not survive after perfusion.

\section{Histology changes in the hippocampus}

\section{Light microscopy}

There was no significant abnormality of tissue structure in different hippocampal regions in the deep hypothermia group, as shown in Figure 1a. There was scattered nerve cell nuclear pyknosis, degredation, and putrescence in the CA I and CA II regions of the hippocampus in normal temperature group, as shown in Figure $1 \mathrm{~b}$.

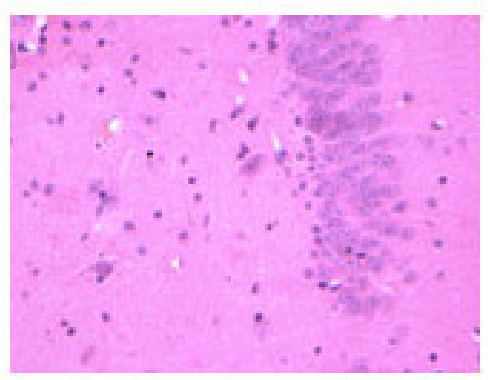

a

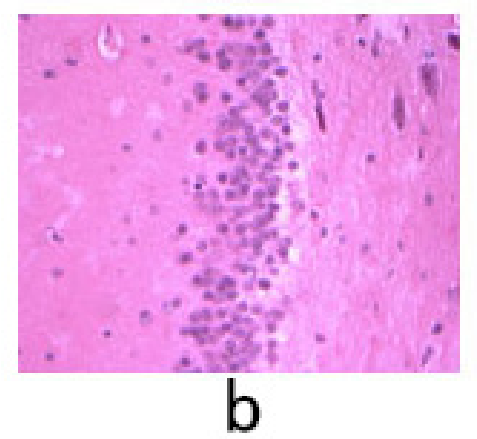

Figure 1. Light microscopic observation of the hippocampus in the two groups (hematoxylin-eosin staining, 400X). a. Hippocompus CAI in the profound hypothermia group. b. Hippocompus CAI in the normothermia group.

\section{Electron microscopy}

In the deep hypothermia group, we observed, under transmission electron microscope, 
that the hippocampal neuronal cell membrane and nuclear membrane were complete with a normal cytoplasm. The mitochondria in the cytoplasm was normal without edema or pyknosis; the endoplasmic reticulum showed a normal shape without extension, as shown in Figure 2a. In the normal temperature group, there was nerve cell edema, endoplasmic reticulum pool

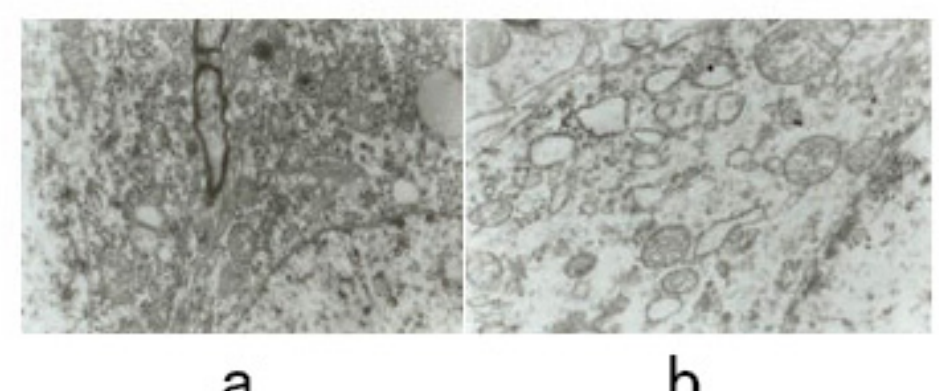

Figure 2. Electron microscopic observation of the hippocampus in the two groups. a. Hippocampus in the profound hypothermia group $(10,000 \mathrm{X})$. b. Hippocampus in the normothermia group $(16,000 \mathrm{X})$.

formation, nerve cell nucleus pyknosis, nerve fiber demyelination, neuropil edema, organelle pathology of mitochondrium puffs, cristae lysis, endoplasmic reticulum extension, and exfoliation grains, as shown in Figure $2 b$.

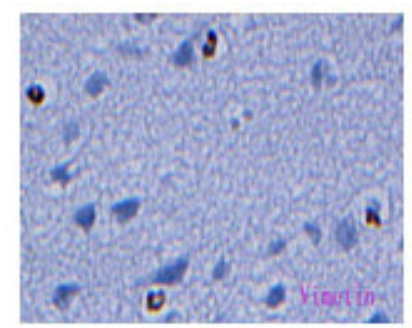

a

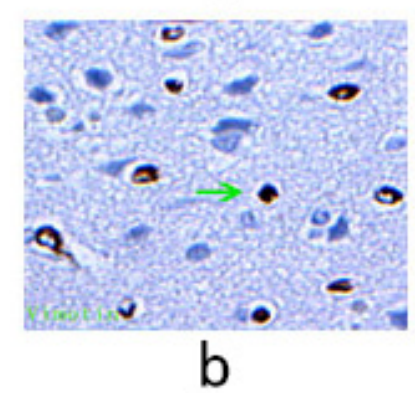

Figure 3. Vimentin expression in the hippocampus of the two groups (400X). a. Normothermia group. b. Profound hypothermia group. 


\section{Vimentin expression}

Vimentin was expressed mainly in the endochylema and was brownish yellow by immunohistochemistry, as shown in Figure 3. The positive rate of vimentin expression in rhesus monkey cerebrum tissue in the deep hypothermia group was $47.88 \% \pm 1.66$, while the rate in the normal temperature group was $79.51 \% \pm 1.00$. There was a statistically significant difference in vimentin expression between the two groups $(\mathrm{P}<0.01)$.

\section{DISCUSSION}

Antegrade cerebral perfusion combined with selective deep hypothermia following severe cerebral ischemia can avoid cardiopulmonary complications that are induced by systemic deep hypothermia, increase local perfusion flow of the pallium, transfer oxygen and electrolytes, remove metabolites, and extend occlusion safe time ( $\mathrm{Pu}$ et al., 2005; Liang et al., 2009). In order to improve clinical practice, the selective cerebral deep hypothermia ischemia recovery model in rhesus monkeys is promoted in this study. Deep hypothermia perfusion for $60 \mathrm{~min}$ was conducted following ischemia induced by bilateral common carotid artery occlusion at normal temperature for $10 \mathrm{~min}$, and 5 rhesus monkeys recovered successfully after $70 \mathrm{~min}$ of ischemia. These results illustrated that there was no obvious abnormality in hippocampus shape or ultrastructure following selective deep hypothermic perfusion after bilateral common carotid artery occlusion, while there were nerve cell edema, pyknosis, nerve fiber demyelination, mitochondrium puffs, cristae lysis, endoplasmic reticulum extension, and exfoliation grains after perfusion at normal temperature.

Vimentin is one of the main intermediate filaments expressed by colloid cells; therefore, it is used as a protein marker for colloid cells. Vimentin exists in both endothelial cells, pericytes originating from the mesoderm, and colloid cells originating from nerve ectoderm. Vimentin is marker of immature astrocyte colloid cells and appears in embryonic stages and two weeks after birth. Its expression is very low in later stages, although it is widely expressed in colloid cells. At the adult stage, vimentin is only expressed in a small number of cells under normal conditions, such as Bergman colloid cells in the encephalon. Vimentin is expressed when various simulations occur, and its expression is enhanced in reactive astrogliosis (Bramanti et al., 2010; Lu et al., 2011). Vimentin is the main cell skeleton in immature colloid cells. It is expressed in a rat cerebral ischemia model where vimentin expression is present in the perinuclear cytoplasm of astrocyte colloid cells and oligodendrocytes. There is increased expression of vimentin during the proliferative response of astrocyte colloid cells after ischemic injury (Shen et al., 2010; Pimentel et al., 2011; Lively et al., 2011). It has been suggested by researches that the distribution of vimentin positive cells are located in neurons surrounding colloid cells, and this distribution is consistent with regions of neuronal necrosis (Joachim et al., 2011; Wurm et al., 2011). After cerebral ischemia, nerve cells will undergo secondary death, resulting in further extension of the infarction zone if apoptosis occurs in astrocyte colloid cells (Perez-Pinzon, 2007). It has been discovered by researchers that vimentin expression induced by ischemia can influence the stability of the cell skeleton (Bargagna-Mohan et al., 2010; Liu et al., 2010). This study has found that selective deep hypothermic perfusion can inhibit increased vimentin expression in the hippocampus after cerebral ischemia.

In conclusion, this study indicated that selective deep hypothermic antegrade cerebral 
perfusion in the rhesus monkey cerebrum after bilateral common carotid artery occlusion for 10 min can reduce damage by cerebral ischemia and protect cerebral tissue.

\section{REFERENCES}

Bargagna-Mohan P, Paranthan RR, Hamza A, Dimova N, et al. (2010). Withaferin A targets intermediate filaments glial fibrillary acidic protein and vimentin in a model of retinal gliosis. J. Biol. Chem. 285: 7657-7669.

Bramanti V, Tomassoni D, Avitabile M, Amenta F, et al. (2010). Biomarkers of glial cell proliferation and differentiation in culture. Front Biosci. 2:558-570.

Cai WQ and Wang BY (1994). Practical Immunocytochemistry and Nucleic Acid Molecule Hybridization Techniques. Ed 1. Cheng Du: Sichuan. 180-191.

Cronberg T, Lilja G, Rundgren M, Friberg H, et al. (2009). Long-term neurological outcome after cardiac arrest and therapeutic hypothermia. Resuscitation 80: 1119-1123.

Elmistekawy EM and Rubens FD (2011). Deep hypothermic circulatory arrest: alternative strategies for cerebral perfusion. A review article. Perfusion 1: 27-34.

Ginsberg MD (2008). Neuroprotection for ischemic stroke: past, present and future. Neuropharmacology 55: 363-389.

Joachim SC, Wax MB, Boehm N, Dirk DR, et al. (2011). Upregulation of antibody response to heat shock proteins and tissue antigens in an ocular ischemia model. Invest. Ophthalmol. Vis. Sci. 52: 3468-3474.

Liang YM, Jiang JY, Mao Q, Zhang S, et al. (2009). Therapeutic window of selective profound cerebral hypothermia for resuscitation of severe cerebral ischemia in primates. J. Neurotrauma 26: 2107-2112.

Liu T, Guevara OE, Warburton RR, Hill NS, et al. (2010). Regulation of vimentin intermediate filaments in endothelial cells by hypoxia. Am. J. Physiol. Cell Physiol. 299: C363-C373.

Lively S, Moxon-Emre I and Schlichter LC (2011). SC1/hevin and reactive gliosis after transient ischemic stroke in young and aged rats. J. Neuropathol. Exp. Neurol. 70: 913-929.

Lu YB, Iandiev I, Hollborn M, Köber N, et al. (2011). Reactive glial cells: increased stiffness correlates with increased intermediate filament expression. FASEB J. 25: 624-631.

Mracek J (2010). Past, present and future of neuroprotection. Cas Lek Cesk. 149: 586-590.

Nakamura K, Nakamura E, Yano M, Niina K, et al. (2011). Factors influencing permanent neurologic dysfunction and mortality after total arch replacement with separate arch vessel grafting using selective cerebral perfusion. Ann. Thorac. Cardiovasc. Surg. 17: 39-44.

Perez-Pinzon MA (2007). Mechanisms of neuroprotection during ischemic preconditioning: lessons from anoxic tolerance. Comp. Biochem. Physiol. A Mol. Integr. Physiol. 147: 291-299.

Pimentel VC, Pinheiro FV, De Bona KS, Maldonado PA, et al. (2011). Hypoxic-ischemic brain injury stimulates inflammatory response and enzymatic activities in the hippocampus of neonatal rats. Brain Res. 1388: 134-140.

Pu J, Zhao XX, Xu W, Feng ZT, et al. (2005). The study of 1H-MRS on monkey of resuscitation after cerebral selection ultra-profound hypothermic blood flow occlusion. Chin. J. Radiol. 39: 1238-1241.

$\mathrm{Pu}$ J, Fu XA, Feng ZT, Jiang T, et al. (2008). Effects of content of glutamate and Gamma-aminobutyric acid of macaca mulattas on resuscitation after selective ultra-profound hypothermia and blood flow occlusion. Chin. J. Neurosurg. 24: 474-476.

Shen CC, Yang YC, Chiao MT, Cheng WY, et al. (2010). Characterization of endogenous neural progenitor cells after experimental ischemic stroke. Curr. Neurovasc. Res. 7: 6-14.

Wurm A, Iandiev I, Uhlmann S, Weidemann P, et al. (2011). Effects of ischemia-reperfusion on physiological properties of Müller glial cells in the porcine retina. Invest. Ophthalmol. Vis. Sci. 52: 3360-3367.

Yao T (2001). Physiology (7 years). Bei Jing: Ren Min Wei Sheng Chu Ban She. 2001: 177. 